\title{
EL CONTROL DE LA LIMPIEZA DE LAS ARENAS POR MEDIO DEL ENSAYO DEL AZUL DE METILENO
}

\author{
(CLEANNESS CONTROL OF THE SANDS BY MEANS OF METHYLENE BLUE TEST)
}

\author{
J. L. Ramirez Ortiz, Prof. Dr. Ing. Industrial \\ J. M. Barcena Diaz, Dr. Ing. Industrial \\ J. I. Urreta Ormaechea, Ing. Industrial \\ LABORATORIOS DE ENSAYOS E INVESTIGACIONES INDUSTRIALES \\ "L. J. Torróntegui» LABEIN (Bilbao)
}

$652-2$

\section{RESUMEN}

Se muestran, en la primera parte del artículo, las líneas fundamentales del trabajo de investigación desarrollado en $L A B E I N$, sobre la "Influencia de los finos calizos y arcillosos de arenas calizas de machaqueo, en las propiedades del hormigón».

Como consecuencia del mismo, se proponen nuevos límites a considerar respecto al contenido admisible de finos en las arenas calizas de machaqueo, llegando a un límite superior del $15 \%$, controlando la naturaleza de los finos por medio del equivalente de arena a vista, $y$, sobre todo, por el Azul de Metileno.

En la segunda parte del artículo, se desarrolla la metodologia utilizada para obtener el Azul de Metileno, en arenas utilizadas en la confección de hormigón, que poseen poca arcilla, basándose en el método aplicado por la normativa francesa, adaptado convenientemente y con diferente expresión de resultados.

\section{SUMMARY}

In the first part of this work, the main lines of the investigation work developed at LABEIN on the ilnfluence of the limy and clayey slimes from calcareous crushed sands on concrete properties" are presented.

As a consequence of it, new limits are proposed to consideration regarding the admissible content of slime in crushed sands, reaching a limit higher than $15 \%$, controling the slime nature by means of an equivalent of normal sand and specially by Methylene Blue.

The second part of the work is devoted to the development of the methodology for the obtention of Methylene Blue, in sands used in the elaboration of concrete which have a little amount of clay, taking as a base the method applied by French normative, duly adapted and with different expression of the results.

\section{INFLUENCIA DE LOS FINOS EN LA CALIDAD DE LAS ARENAS}

\subsection{Introducción}

La limpieza de las arenas es un problema antiguo, que no se ha logrado ver reflejado todavia satisfactoriamente, en la normativa española. La Instrucción EH-82 establece que el contenido de particulas finas (menores de 80 micras) ha de ser inferior al $5 \%$.

En los laboratorios LABEIN, conscientes desde hace muchos años de la problemática que sobre todo las arenas calizas de machaqueo tienen a este respecto, se ha llevado a cabo un trabajo de investigación plurianual cuya última etapa trata de la influencia de estas particulas finas en las propiedades del hormigón (3).

Previamente, se habia desarrollado dentro del proyecto un estudio sobre las canteras del Pais Vasco (1), y otro sobre la influencia de los finos en los morteros (2).

A lo largo del proyecto de investigación se constata, que no son los finos calizos los causantes de las bajas resistencias, sino que este efecto es debido fundamentalmente a la presencia de finos de naturaleza arcillosa existentes en las arenas.

El propósito de este articulo no es presentar los resultados de esta última etapa del proyecto contenidos en la monografia (3), lo que es nuestra intención hacer en un futuro próximo con suficiente amplitud, sino exponer brevemente sus conclusiones, que posiblemente influyan en el cambio de la $\mathrm{EH}-82$ en este tema, para después explicar con detalle el método operativo de un ensayo que llevará 
aparejado la nueva normativa y que ha sido experimentado y adaptado en nuestro programa de investigación.

\subsection{Planteamiento del estudio de la influencia de los finos calizos y arcillosos sobre hormigones de caliza triturada}

A partir de las conclusiones obtenidas trabajando sobre morteros (2), se definieron las lineas básicas de actuación con las arenas utilizadas en la confección de hormigones (3).

Establecida una granulometria básica, se ha hecho variar el contenido de finos totales de la arena desde 3,5 a $25 \%$. Asimismo, se han preparado muestras con un recorrido del contenido de arcilla en la arena desde 0 a $4 \%$. El conjunto de arenas utilizadas se presentan en la tabla n.o 1.

Tabla 1

\begin{tabular}{|c|c|c|c|c|}
\hline \multirow{2}{*}{$\begin{array}{l}\text { Finos totales } \\
\text { en la arena } \\
f\left(\frac{0}{0}\right)\end{array}$} & \multicolumn{4}{|c|}{$\frac{\text { Arcilla }}{\text { Arena }}=i \quad(\%)$} \\
\hline & 0 & 1 & 2 & 4 \\
\hline 25 & * & * & * & * \\
\hline 20 & * & * & * & * \\
\hline 15 & * & * & * & * \\
\hline 10 & * & * & * & * \\
\hline 5 & * & * & * & \\
\hline 3,5 & * & * & & \\
\hline
\end{tabular}

Los finos totales de esta tabla están compuestos de finos calizos puros y finos arcillosos, si los hubiere.

Se han utilizado dos dosificaciones en cemento: 250 y $325 \mathrm{~kg} / \mathrm{m}^{3}$.

Con un guijillo $(5 / 12)$ y un guijo $(12 / 25)$ de árido calizo machacado, haciendo variar las arenas definidas en la tabla n.o 1 , se han preparado las masas de hormigón, todas ellas con la misma manejabilidad para cada dosificación, definidas por el maniabilimetro del L.C.P.C. de Paris.

Con cada variante de la arena y las masas con ellas confeccionadas, se ha realizado la siguiente serie de ensayos:

- Definición de la arena:

- Equivalente de arena (a vista y a pistón)

- Contenido en finos

- Azul de Metileno
- Plasticidad de las amasadas:

- Cono de Abrams

- Maniabilimetro

- Probetas de hormigón:

- Resistencia a compresión

- Resistencia a tracción

- Módulo de elasticidad

- Densidad aparente

- Absorción de agua

- Rebote con el esclerómetro Schmidt

- Velocidad de propagación de ultrasonidos

\subsection{Control de las sustancias perjudiciales}

Habitualmente, se ha considerado que las partículas inferiores a 80 micras son perjudiciales. Con el fin de evaluar su incidencia se ha medido el contenido en finos de todas las arenas de acuerdo a la norma UNE 7-135-58.

Asimismo se ha realizado el ensayo del equivalente de arena, con el método de pistón siguiendo la norma UNE 7-324-76,

habitualmente utilizado para la caracterización de materiales destinados a la construcción de carreteras y puentes (PG3). Igualmente se ha calculado el valor del equivalente de arena a vista, siguiendo la norma francesa NF P 18-598, que se utiliza para determinar la limpieza de las arenas naturales destinadas a la fabricación de hormigón, NF P 18-301.

Exigido tambièn por esta última norma, se ha llevado a cabo el ensayo del Azul de Metileno de acuerdo a la norma experimental P 18-592 (método de la mancha). Los resultados de este ensayo los hemos presentado bajo dos formas:

- AM (g de Azul/100 g de finos)

- $A M^{*}$ (g de Azul/100 g de arena)

Considerando los resultados obtenidos para el equivalente de arena a vista, que da unos valores ligeramente superiores a los de pistón, hemos observado que para valores próximos a 65 , exigido en la normativa francesa, se produce una gran permisividad para arenas de bajo contenido en finos totales y alto en arcilla que dan lugar a bajas resistencias. Si el limite del equivalente de arena se eleva a 75 , se pueden llegar a eliminar arenas de elevado contenido en finos pero carentes de arcilla que dan sin embargo buenas resistencias. La capacidad de discriminación del equivalente de arena es semejante a la del contenido en finos y por lo tanto nos parece que pierde sentido su utilización conjunta. Por otra parte, Lhorty (4) ha 
demostrado que este ensayo presenta una gran dispersión con la granulometria de la muestra, etc.

Como contrapartida, el equivalente de arena es muy conocido y empleado en los laboratorios de obra civil, aspecto éste que no presentan otros ensayos como el Azul de Metileno.

En cuanto a los ensayos de Azul de Metileno realizados, la primera de las expresiones ( $\mathrm{g}$ Azul/100 g finos) es la utilizada por la normativa francesa. La segunda (g Azul/100 g arena), a la vista de los resultados obtenidos (3) opinamos se relaciona más fielmente con la variación de resistencia al incrementar el contenido de finos calizos y/o arcillosos. Se ha observado que para un nivel de arcilla determinado, al incrementar los finos totales, el Azul de Metileno expresado en la forma g de Azul/100 g arena aumenta de una forma lineal aunque suave, disminuyendo lentamente la resistencia. Por otra parte, un incremento de arcilla supone un decremento importante de la resistencia y un incremento claro del Azul de Metileno.

Por el contrario, bajo la expresión g Azul/100 g finos, al incrementar la arcilla, aumenta el Azul de Metileno, mientras que en cada nivel de arcilla, al aumentar los finos la exigencia de Azul de Metileno disminuye siguiendo una tendencia semejante a una hipérbola de expresión AM.f = cte. Este tipo de expresión no se relaciona directamente con el fenómeno resistente.

Por lo tanto, se puede extraer que el Azul de Metileno, bajo la forma g Azul/100 g arena, se corresponde mejor, que bajo la expresión $\mathrm{g}$ Azul/100 g finos, con la evolución de resistencia al variar el contenido de arcilla y/o el de finos. La correspondencia es de sentido inverso.

Gráfico 1. - Obras ordinarias Dosificación $325 \mathrm{~kg} / \mathrm{m}^{3}$

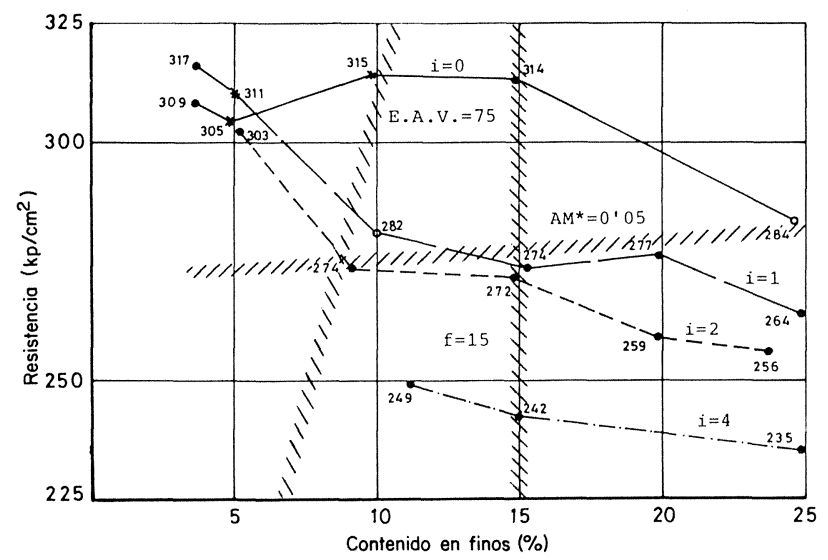

\subsection{Consideración de nuevos límites de finos en arenas calizas de machaqueo}

A la vista de los resultados medios obtenidos en los diferentes ensayos realizados sobre probetas de hormigón, y las arenas utilizadas en su confección (3), se ha llegado a la siguiente propuesta de nuevos limites a considerar para las arenas calizas de machaqueo destinadas a la fabricación de hormigón.

\section{- OBRAS ORDINARIAS}

Condiciones a cumplir:

A) Contenido en finos (\%): $\leqslant 15$

B) Equivalente de arena a vista

B.1) E.A.V. ₹ 75: Aceptable

B.2) E.A.V. < 75: Realizar el ensayo del Azul de Metileno (g Azul/100 g arena)

A.M. $\leqslant$ 0,05: Aceptable

A.M. > 0,05: Rechazable

\section{- OBRAS EN AMBIENTE MAS RIGUROSO}

Condiciones a cumplir:

A) Contenido en finos (\%): $\leqslant 10$

B) Equivalente de arena a vista

B.1) E.A.V. $\geqslant 80:$ Aceptable

B.2) E.A.V. < 80: Realizar el ensayo del Azul de Metileno (g de Azul/100 g arena)

A.M. $\leqslant$ 0,03: Aceptable

A.M. > 0,03: Rechazable

En los gráficos 1, 2, 3 y 4, quedan representadas las lineas significativas correspondientes a las limitaciones anteriores, sobre los resultados de la resistencia a compresión de probetas cilindricas normalizadas ensayadas a la edad de 28 dias.

Gráfico 2. - Obras ordinarias Dosificación $250 \mathrm{~kg} / \mathrm{m}^{3}$

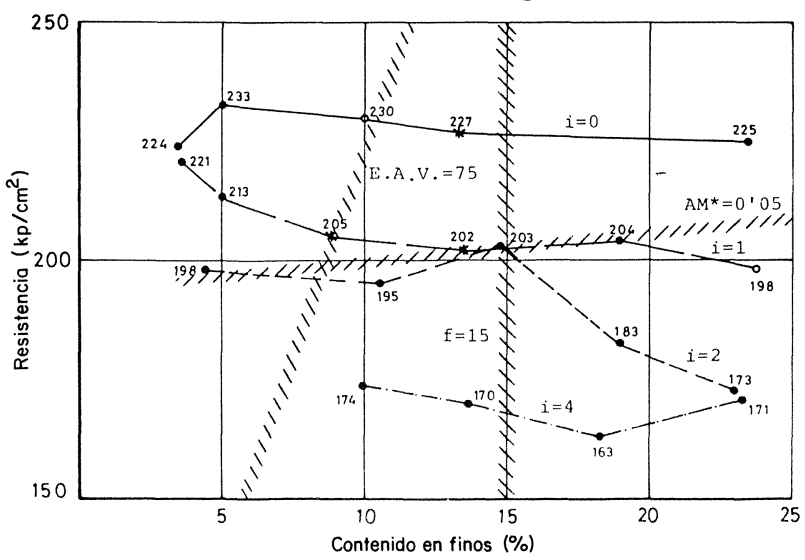


Gráfico 3. - Obras en ambiente más riguroso Dosificación $325 \mathrm{~kg} / \mathrm{m}^{3}$

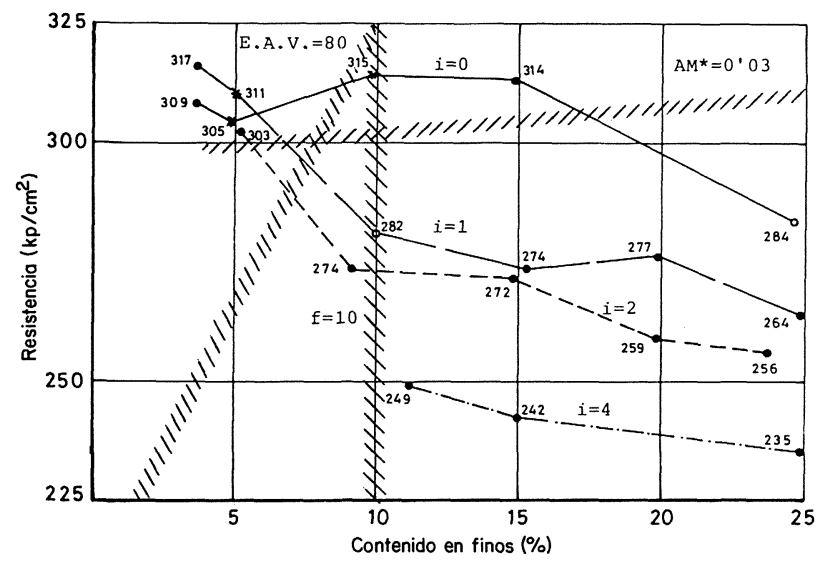

La justificación de los limites de esta propuesta viene desarrollada en la monografia de LABEIN: "Influencia de los finos calizos y arcillosos de arenas calizas de machaqueo, en las propiedades del hormigón” (3) y se refiere, exclusivamente, como se ha dicho en varias ocasiones a arenas provinientes de trituración de calizas.

La presente propuesta exige realmente una exposición y profundización a partir de los resultados de todo el programa de trabajo realizado, cosa que nos proponemos hacer en una próxima ocasión. En este momento se ha querido plantear sin mayor justificación al objeto de poner el énfasis en la potencialidad y método operativo del ensayo del Azul de Metileno.

\section{EL ENSAYO DEL AZUL DE METILENO}

\subsection{Generalidades}

Este ensayo mide la fracción arcillosa activa que el material es susceptible de aportar, por medio de una valoración de la superficie del conjunto de dichas partículas, lo que depende de la naturaleza y de la proporción de la arcilla en la muestra. Es por lo tanto una medida global de la polución arcillosa tanto en cantidad como en actividad (5).

El ensayo consiste en determinar la cantidad de Azul de Metileno necesario para recubrir de una pelicula monomolecular la superficie total, externa + interna, de las partículas arcillosas de un material mantenido en suspensión en el agua (6).

Los finos calizos también absorben Azul de Metileno aunque en mucha menor proporción que los arcillosos (3) y (5).
Gráfico 4. - Obras en ambiente más riguroso Dosificación $250 \mathrm{~kg} / \mathrm{m}^{3}$

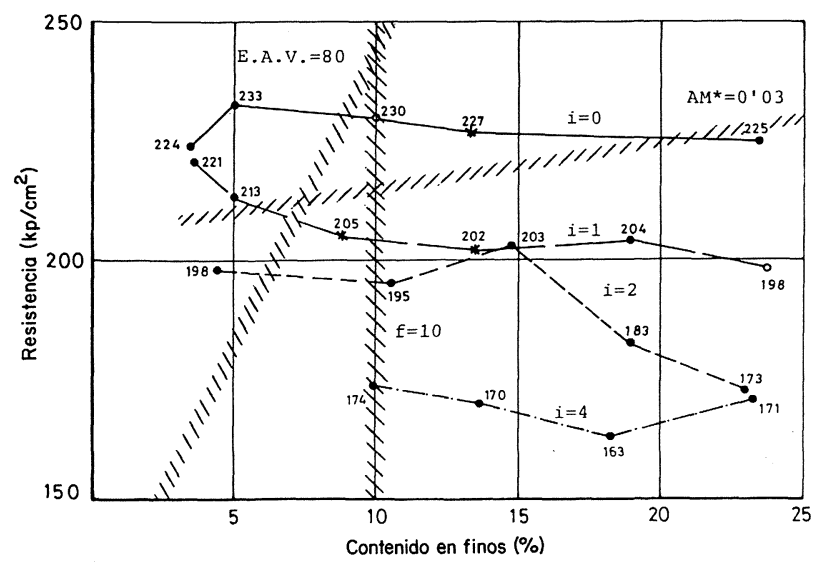

En los últimos meses, con la colaboración del Centro de Investigación y Desarrollo de Cementos Asland (Toledo), y el Departamento de Materiales de la Escuela Técnica Superior de Ingenieros de Caminos, Canales y Puertos de Barcelona se ha puesto a punto, mediante el oportuno contraste de resultados, el siguiente método operativo del ensayo del Azul de Metileno. Se ha de resaltar que no se ha conseguido, por el momento, una aceptable correlación del método de la mancha, descrito en lo que sigue, con el del turbidimetro, normalizado según AFNOR P 18-595.

\subsection{Método operativo}

El método operativo se ha basado en la norma experimental AFNOR P 18-592, de julio de 1980, y en los trabajos previos desarrollados en el Laboratoire Central des Ponts et Chaussées de Paris (7).

En las arenas destinadas a la confección de hormigón, la cantidad de particulas arcillosas es habitualmente más bien escasa, y ello tiene como consecuencia que con poca cantidad de Azul de Metileno se finaliza el ensayo, y por lo tanto el método pierde precisión. Para aumentar esta precisión, es necesario incrementar la cantidad de arcilla de la muestra a ensayar, y/o disminuir la cantidad de Azul que se introduce en la muestra en cada inyección.

El método operatorio que se propone, después de la oportuna consideración de las variables en juego y las pruebas realizadas es el siguiente:

\section{A) PREPARACION DE LA MUESTRA}

\section{A.1) Arenas muy contaminadas}

- Cuartear una muestra de arena (0/5), entre 300 y 400 gramos. 
- Secar a peso constante (M).

- Introducir la muestra en un vaso de precipitados de 1 litro de capacidad y añadir 500 c.c. de agua destilada.

- Dejar reposar 24 horas.

\section{A.2) Arenas poco contaminadas}

- Cuartear una muestra de arena entre 1000 y 1500 gramos.

- Secar a peso constante (M).

- Tamizar en seco sobre el tamiz de luz de malla $0,59 \mathrm{~mm}$. El material que pasa puede llegar hasta los $400 \mathrm{~g}$; si no es asi y se desea una mayor precisión, se puede incrementar la cantidad de la muestra original.

- El material retenido por el tamiz de 0,59 mm de luz de malla se tamiza por via húmeda sobre este mismo tamiz, utilizando la misma metodologia que la empleada para obtener el contenido en finos de una muestra (UNE 7135): "hasta que el agua que sale del tamiz esté totalmente exenta de partículas en suspensión". El liquido con las partículas en suspensión se recoge en un vaso de precipitados de capacidad suficiente.

- Dejar decantar las partículas y eliminar el agua sobrante. Introducir el material del fondo en otro vaso de precipitados de base más estrecha de 1 litro de capacidad, y repetir la operación hasta conseguir eliminar la mayor parte del agua.

- Añadir a estas partículas obtenidas por vía húmeda, el material conseguido por tamizado en seco.

- Completar el liquido hasta aproximadamente 500 c.c., utilizando agua destilada.

- Dejar reposar 24 horas.

En la foto 1 se muestra el material resultante del tamizado en seco introducido en el vaso pequeño, asi como el liquido, junto con las partículas en suspensión, obtenida de la limpieza de la muestra de arena ya tamizada,

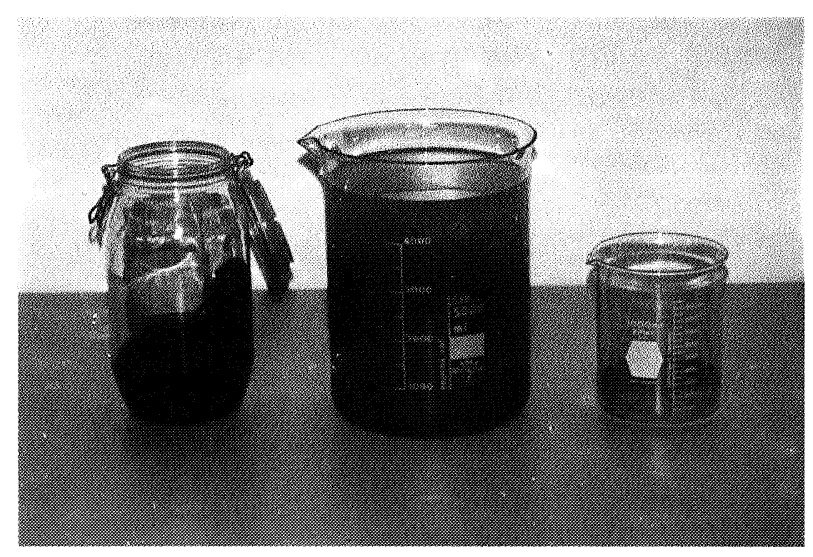

Foto 1

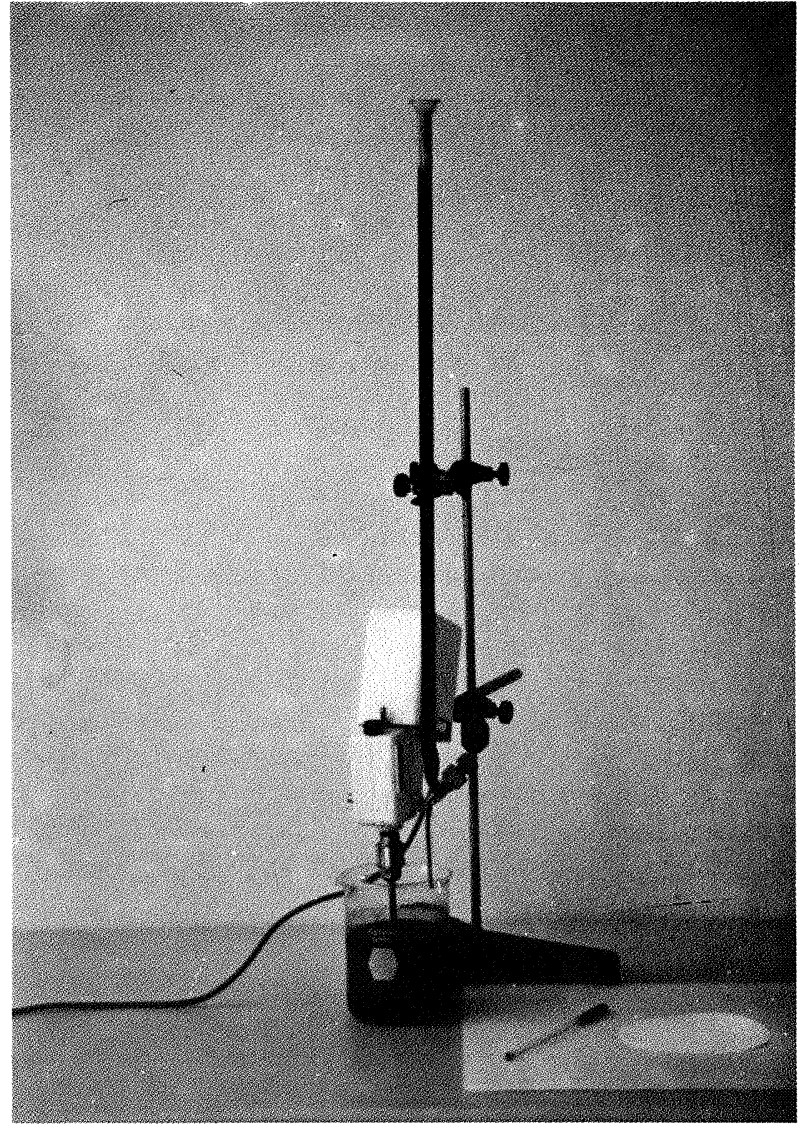

Foto 2

sobre el tamiz de $0,59 \mathrm{~mm}$ de luz de malla, en el vaso de 5 litros.

Al final del proceso todas las partículas inferiores a 0,59 mm se encuentran en el vaso de 1 litro de capacidad, donde se realiza posteriormente el ensayo.

\section{B) ENSAYO DE LA MANCHA}

\section{B.1.) Aparatos necesarios (Foto 2):}

- Una bureta

- Papel de filtro, del tipo WHATMAN n.o 40.

- Varilla de vidrio, de $8 \mathrm{~mm}$ de diámetro.

- Agitador de aletas.

- Recipiente de 1 litro de capacidad; diámetro alrededor de $100 \mathrm{~mm}$.

- Un cronómetro.

\section{B.2) Producto:}

- Solución de Azul de Metileno. Concentración: $10 \mathrm{~g} \pm 0,1 \mathrm{~g} /$ litro.

\section{B.3.) Ensayo:}

Después de cada inyección de Azul, el ensayo consiste en obtener una gota de suspensión por 


\section{Gráfico 5}

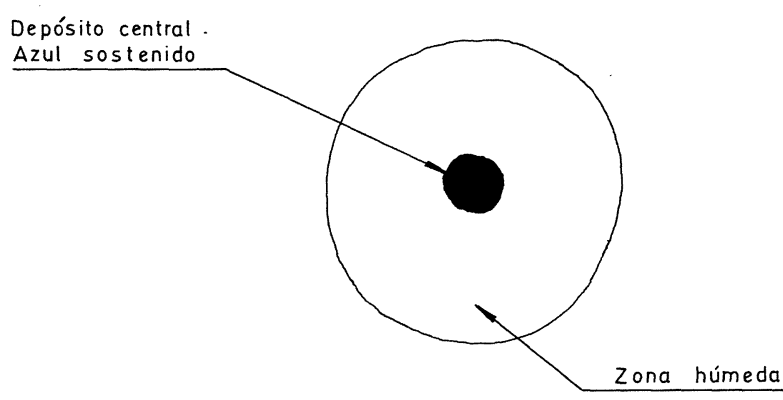

NEGATIVO

Depósito central Azul sostenido

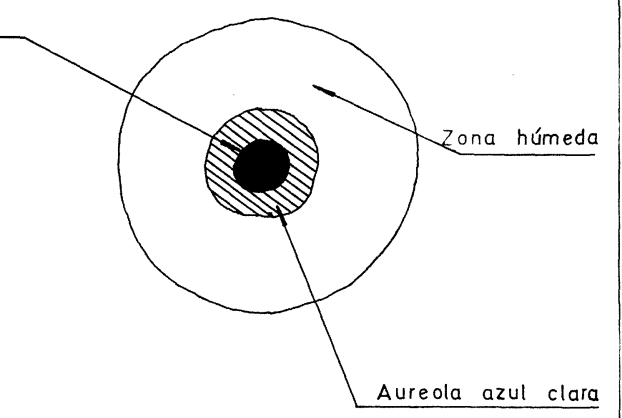

POSITIVO

Gráfico 6. - Esquema de dosificación

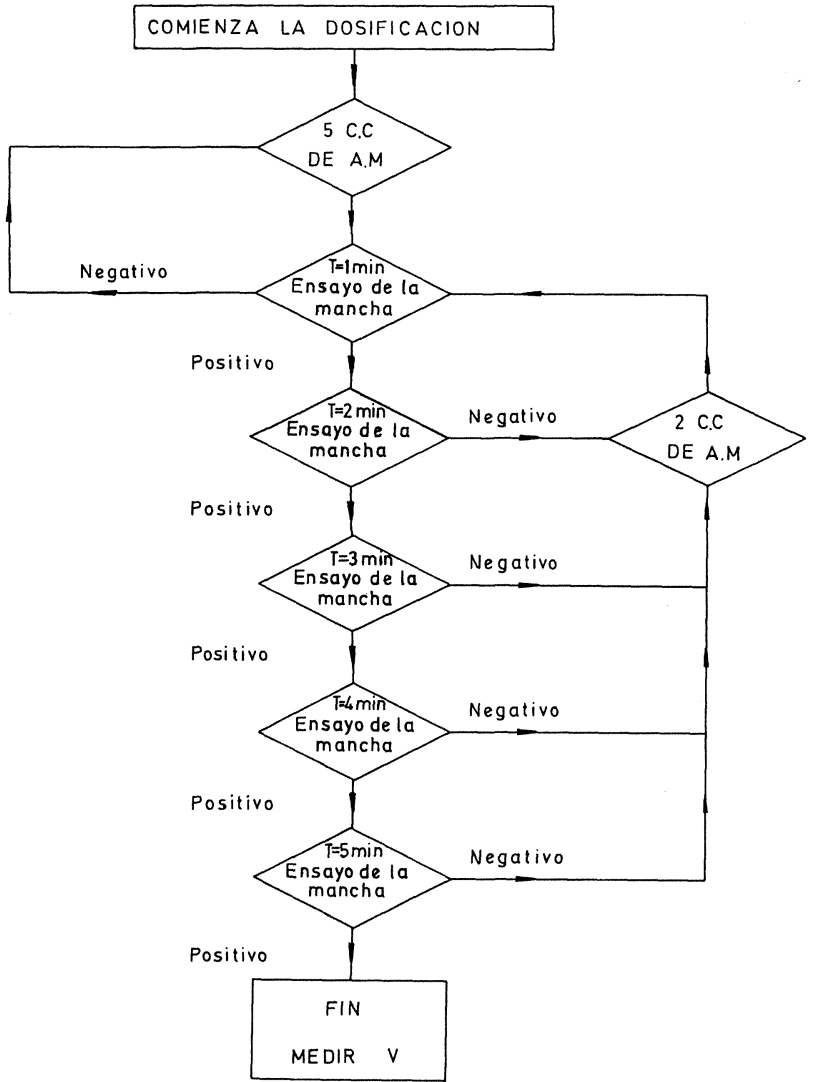

medio de la varilla y depositarlo en el papel de filtro. La mancha se compone de un depósito central de material coloreado de un azul sostenido, rodeado de una zona húmeda.

El test es positivo si en la zona húmeda aparece, alrededor del depósito central una aureola azul clara; si la aureola es incolora el test es negativo. Ver gráfico 5 .

El proceso de dosificación del Azul de Metileno se lleva a cabo de acuerdo con el esquema que se presenta en el gráfico 6 .

Cada adición de Azul es seguida de ensayos de la mancha efectuados siempre de minuto en minuto. Hay que repetir las operaciones de adición y ensayo hasta que se consiga que el test se mantenga positivo durante cinco minutos consecutivos. Entonces, la dosificación se considera terminada y se lee el volumen $\mathrm{V}$ empleado de Azul de Metileno.

A lo largo de todo el ensayo la muestra ha de estar en movimiento. Para ello el agitador, antes de empezar la dosificación, debe agitar la muestra durante 1 minuto a una velocidad de 700 r.p.m., y el resto del ensayo a 400 r.p.m. Estos valores son orientativos, siendo variables en función de la granulometria y de la masa de la muestra. En el caso de que se necesite una mayor precisión se puede disminuir la dosificación de Azul de Metileno desde el comienzo del ensayo a la cantidad de 2 c.c. para cada adición

El resultado final se expresa de la siguiente forma:

A.M. $=\frac{V}{M}$

A. M: Azul de Metileno (g Azul/100 g arena)

V: Volumen de Azul en c.c. (10 g/litro)

M: Peso de la muestra seca en gramos.

Ahora bien, si se desea comprobar si una arena cumple la propuesta de norma expresada anteriormente, basta inyectar la cantidad de azul que define el limite de aceptación.

\section{OBRAS ORDINARIAS}

$\mathrm{AM}=\frac{\mathrm{V}}{\mathrm{M}} \leqslant 0,05$

$V=0,05 M$

OBRAS EN AMBIENTE MAS RIGUROSO

$A M=\frac{V}{M} \leqslant 0,03$

$V=0,03 M$ 
El volumen resultante de esta operación se añade de una sola vez, efectuándose el ensayo de la mancha después de 8 minutos de agitación:

- Si el ensayo es positivo (aureola azul clara), existe Azul de Metileno por exceso o estamos en el limite; por lo tanto la arena se aceptaria.

- Si el ensayo es negativo (aureola incolora), la muestra admite mayor cantidad de Azul; por lo tanto la arena se rechazaria.

Ha de procederse a la limpieza del material utilizado una vez que se haya finalizado el ensayo. Los depósitos de Azul de Metileno se limpian bien con agua si son recientes.

\section{BIBLIOGRAFIA}

1. RAMIREZ, J. L.; BARCENA, J. M; URRETA, J. I.: "Estudio sobre la nocividad y corrección de los finos de las arenas calizas de machaqueo para hormigones, en el Pais Vasco (1.a Fase: Prospección de Canteras)"

Monografia LABEIN: 1/I.100.81 - septiembre 1983.
2. RAMIREZ, J. L.; BARCENA, J. M.; URRETA, J. I.: "Influencia de los finos calizos y arcillosos en la resistencia de morteros de cemento". Monografía LABEIN: 15/I.100.81 - octubre 1984.

3. RAMIREZ, J. L.; BARCENA, J. M.; URRETA, J. I.: "Influencia de los finos calizos y arcillosos de arenas calizas de machaqueo, en las propiedades del hormigón».

Monografia LABEIN: 31/I.100.81 - mayo 1986.

4. LHORTY: "Considèrations sur l'equivalent de sable" Revue général des routes et aérodromes, n.o 289, febrero, 1956.

5. TRAN NGOC LAN: “L'essai au Bleu de Méthylène. Un progrès dans la mesure et le contrôle de la propreté des granulats". Bulletin Liaison des Laboratoires des Ponts et Chaussées, n.o 107, mayo-junio 1980.

6. ZILMAR R. UNIKOWSKI: “Influence des argiles sur les propriétés des mortiers de ciment». Rapport de recherche LPC n.o 110. Laboratoire Central des Ponts et Chaussées, febrero 1982.

7. TRAN NGOC LAN: “L'essai au Bleu de Méthylène. Avantprojet de mode operatoire». Laboratoire Central des Ponts et Chaussées, octubre 1979.

\section{publicación del i.e.t.c.c.}

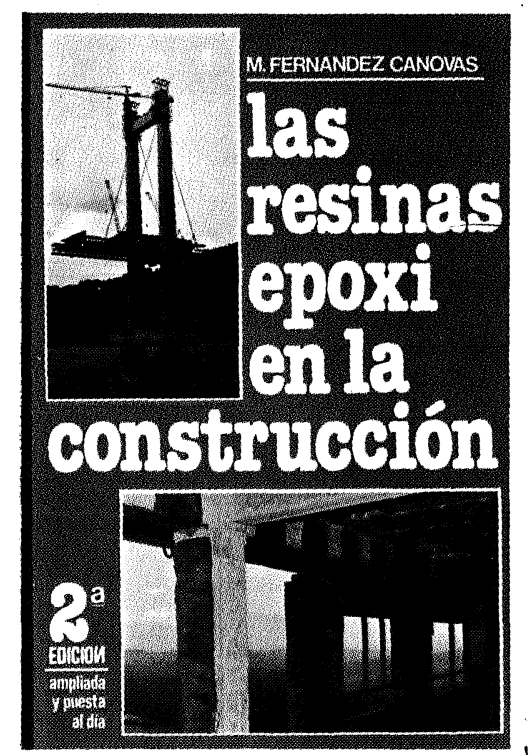

Manuel Fernández Cánovas

Dr. Ingeniero de Construcción

Este libro, el primero en lengua castellana sobre resinas epoxi aplicadas a la construcción, está dirigido a arquitectos, ingenieros, constructores y aplicadores. En él, sobre una reducida base teórica imprescindible, se asienta toda una extensa gama de aplicaciones de gran interés.

El autor trabaja desde hace muchos años en el campo de la investigación, especialmente en el estudio de refuerzos y reparaciones estructurales realizados con resinas epoxi.

Con un lenguaje sencillo se tocan todos los problemas que pueden presentarse en la construcción $y$ en los que la solución puede radicar en el correcto empleo de las resinas epoxi.

Se estudian los componentes de las formulaciones epoxi, sus propiedades fisicas y quimicas, y aplicaciones, deteniéndose, detalladamente, en las siguientes:

Unión de hormigón fresco a hormigón endurecido. - Unión de hormigones entre si.-Inyecciones de fisuras y grietas. - Unión de acero a hormigón. - Barnices y pinturas. - Las combinaciones brea-epoxi. - Revestimientos de depósitos alimenticios. - Sellado de superficies cerámicas. - Protección de tubos. - Los suelos epoxi en sus diferentes variantes. - Terrazo epoxi. - Reparación de baches. - Reparación de desperfectos en estructuras. - Reparación vigas, fojados y zapas, etc. Consolidacicas. Gur. Gre

Se termina con unos capitulos dedicados a la limpieza y preparación de las superficies según los materiales a unir; al control del estado superficial de éstos; a las condiciones de temperatura de aplicación; limpieza de los útiles de

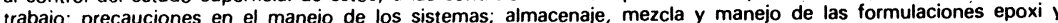
métodos de ensayo de sistemas y aplicaciones epoxidicas.

Un volumen encuadernado en cartoné plastificado con lomo de tela, de $17 \times 24 \mathrm{~cm}$, compuesto de 334 páginas y 158 figuras y fotografias.

Madrid. 1981

Precios: España, 1.700 ptas.; extranjero, \$ USA 34.00. 